\title{
Coma reversal after basilar artery thrombolysis
}

\author{
Lee I. Kubersky, MD; Andreas Kramer, MD; and Bradford B. Worrall, MD, MSc
}

A 36-year-old woman without prior medical problems presented 45 minutes after collapsing. Examination revealed coma with asymmetric unreactive pupils, dysconjugate gaze, absent oculocephalic reflex, and minimal reflexive movements. Her initial National Institutes of Health Stroke Scale (NIHSS) score was 24.

Noncontrasted head CT and CT angiogram (figure 1) confirmed basilar artery occlusion (BAO). IV recombinant tissue plasminogen activator (IV rt-PA) was given 2 hours after symptom onset. Posttreatment cerebral angiography revealed a patent basilar artery and MRI demonstrated
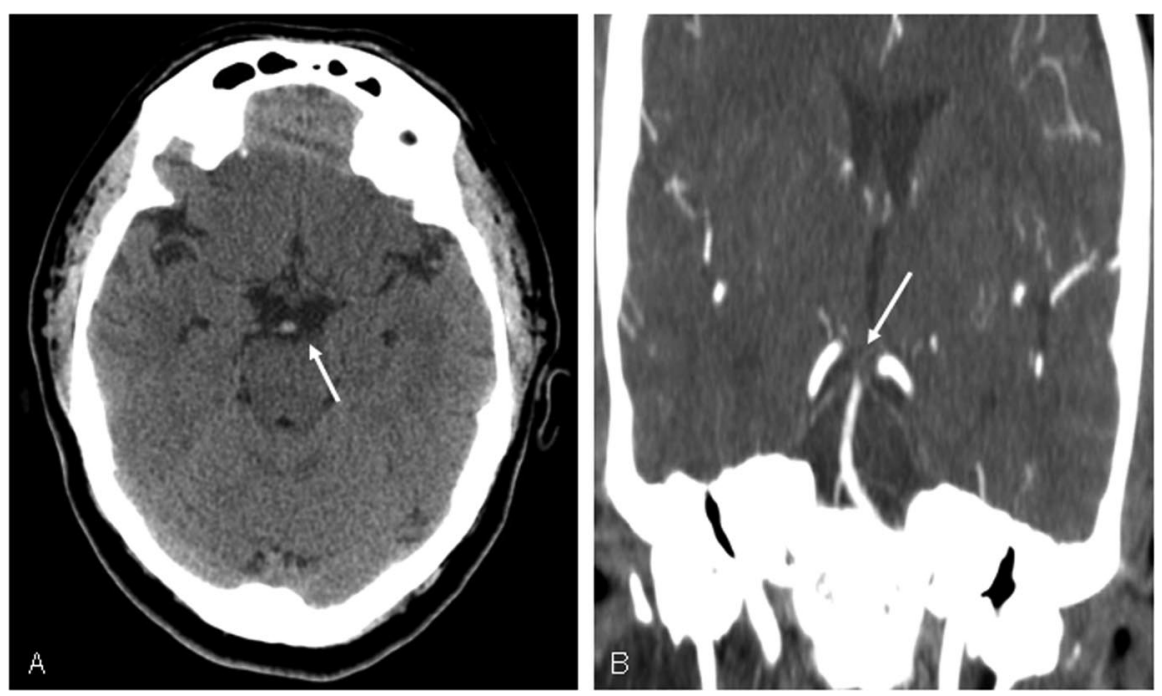

Young age, short occlusion, and recanalization are associated with increased survival after $\mathrm{BAO},{ }^{2}$ as evidenced by our patient. The additional time required for IA therapy remains to be justified. Other strategies, including a combined IV/IA bridging regimen, need to be rigorously investigated. pital with minimal neurologic deficits, which included an incomplete left homonymous hemianopsia. Total NIHSS score at discharge was 3.

Optimal treatment for BAO is controversial. Intra-arterial (IA) rt-PA results in better recanalization rates, but outcomes may not be better than IV rt-PA. ${ }^{1}$

\section{References}

1. Lindsberg PJ, Mattle HP. Therapy of basilar artery occlusion: a systematic analysis comparing intra-arterial and intravenous thrombolysis. Stroke 2006;37:922-928.

2. Brandt T, von Kummer R, Muller-Kuppers M, Hacke W. Thrombolytic therapy of acute basilar artery occlusion: variables affecting recanalization and outcome. Stroke 1996;27: 875-881.

Figure 1. (A) Unenhanced cranial $C T$ showing relative hyperdense basilar artery as compared to the middle cerebral arteries signifying acute thrombosis. (B) CT angiogram, coronal section showing absence of flow in distal basilar artery. 


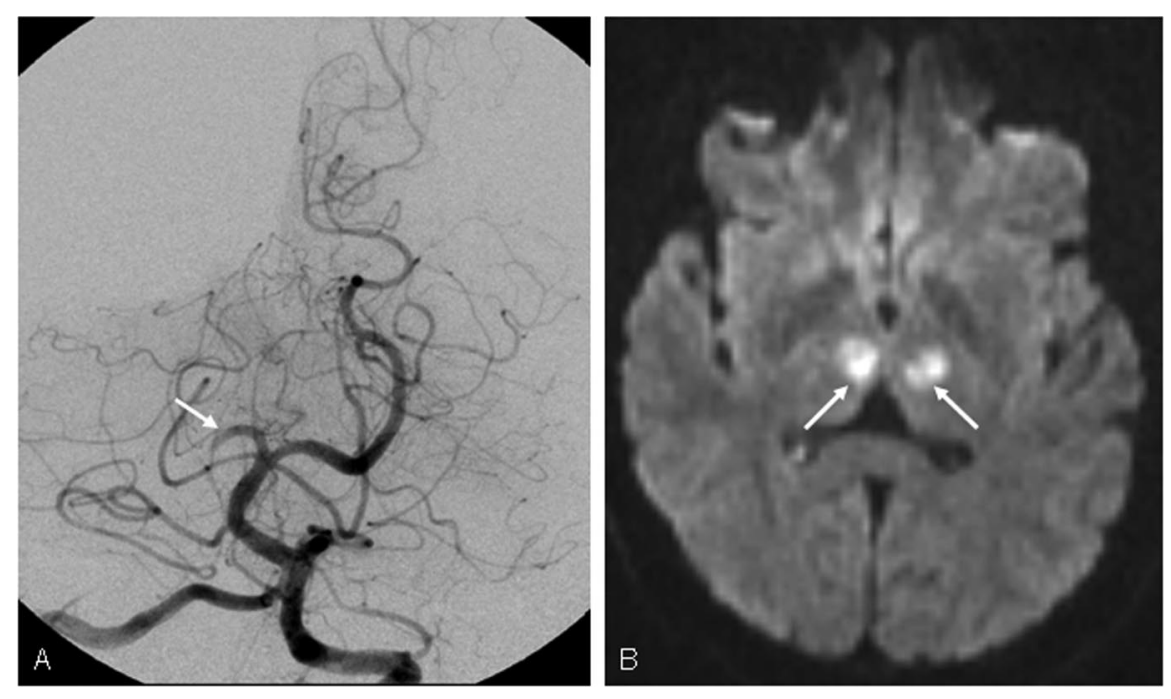

Figure 2. (A) Cerebral angiogram showing patent basilar artery 3 hours after thrombolysis with migrated clot in proximal right posterior cerebral artery. (B) Diffusionweighted image MRI sequence performed at 8 hours demonstrated bilateral thalamic infarcts. MRI also demonstrated scattered infarctions in the right posterior cerebral artery and right superior cerebellar artery territories (not shown). 


\title{
Neurology
}

\author{
Coma reversal after basilar artery thrombolysis \\ Lee I. Kubersky, Andreas Kramer and Bradford B. Worrall \\ Neurology 2007;68;E11-E12 \\ DOI 10.1212/01.wnl.0000256814.55729.7e
}

This information is current as of March 12, 2007

\section{Updated Information \& Services}

\section{References}

\section{Subspecialty Collections}

Permissions \& Licensing

Reprints including high resolution figures, can be found at: http://n.neurology.org/content/68/11/E11.full

This article cites 2 articles, 2 of which you can access for free at: http://n.neurology.org/content/68/11/E11.full\#ref-list-1

This article, along with others on similar topics, appears in the following collection(s):

\section{All Cerebrovascular disease/Stroke}

http://n.neurology.org/cgi/collection/all_cerebrovascular_disease_strok $\mathrm{e}$

\section{All Clinical trials}

http://n.neurology.org/cgi/collection/all_clinical_trials Clinical trials Observational study (Cohort, Case control)

http://n.neurology.org/cgi/collection/clinical_trials_observational_study _cohort_case_control

Infarction

http://n.neurology.org/cgi/collection/infarction

Information about reproducing this article in parts (figures,tables) or in its entirety can be found online at:

http://www.neurology.org/about/about_the_journal\#permissions

Information about ordering reprints can be found online:

http://n.neurology.org/subscribers/advertise

Neurology ${ }^{\circledR}$ is the official journal of the American Academy of Neurology. Published continuously since 1951, it is now a weekly with 48 issues per year. Copyright . All rights reserved. Print ISSN: 0028-3878. Online ISSN: 1526-632X.

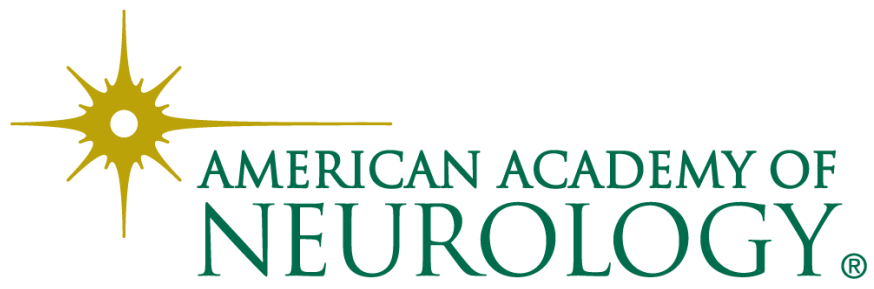

